\title{
Optimizing antimicrobial prescription through e-health: setting, dosing, timing and stewardship
}

\author{
C Palos", A Bispo, P Rodrigues, L França, M Capoulas, ICAC and Infection Control and Antibiotics Committee \\ From 3rd International Conference on Prevention and Infection Control (ICPIC 2015) \\ Geneva, Switzerland. 16-19 June 2015
}

\section{Introduction}

Antimicrobial resistance is a major issue of of healthcare and new resistances are being observed. Antibiotics should be used strictly. When prescribed, they should be selected in accordance to a diagnosis, epidemiology and other factors, requiring appropriate dosing and duration of treatment. Antibiotic prescription should be seen as a team decision, where antimicrobial stewardship plays a major role. E-health, defined as the intensive use of information and communication technologies in the health sector, should be maximized in order to help physicians in prescribing right antibiotics on the right contexts, dosing and duration.

\section{Objectives}

To optimize global antimicrobial prescription in a paper free hospital.

\section{Methods}

A new template for antimicrobial prescription was created, allowing visualization and printing of first line guidelines. When prescribing antimicrobials, context is firstly requested: surgical prophylaxis, therapeutics, others (droplists for all). In doing so, the usual dose and the maximum duration for the selected context automatically appear by default ( 1 day for SSI prophylaxis; variable days for each infection, 7 days for the majority). When the selected antimicrobial is not matched and/or it belongs to a group of conditioned use (quinolones, carbapenems, vancomycin, e.g.), a pop-up message appears on the screen and justification is mandatory. An automated e-mail with those details is immediately sent to a subgroup of the ICAC and Pharmacy.

Hospital Beatriz Ângelo Loures Portugal 